\title{
A fractional counting process and its connection with the Poisson process
}

\author{
Antonio Di Crescenzo, Barbara Martinucci and Alessandra Meoli \\ Dipartimento di Matematica \\ Università degli Studi di Salerno \\ Via Giovanni Paolo II n. 132, \\ 84084 Fisciano (SA), Italy. \\ E-mail address: \{ adicrescenzo, bmartinucci, ameoli \} Qunisa.it
}

\begin{abstract}
We consider a fractional counting process with jumps of amplitude $1,2, \ldots, k$, with $k \in \mathbb{N}$, whose probabilities satisfy a suitable system of fractional difference-differential equations. We obtain the moment generating function and the probability law of the resulting process in terms of generalized Mittag-Leffler functions. We also discuss two equivalent representations both in terms of a compound fractional Poisson process and of a subordinator governed by a suitable fractional Cauchy problem. The first occurrence time of a jump of fixed amplitude is proved to have the same distribution as the waiting time of the first event of a classical fractional Poisson process, this extending a well-known property of the Poisson process. When $k=2$ we also express the distribution of the first passage time of the fractional counting process in an integral form. Finally, we show that the ratios given by the powers of the fractional Poisson process and of the counting process over their means tend to 1 in probability.
\end{abstract}

\section{Introduction and background}

Fractional Poisson processes and related counting processes are attracting the attention of several authors. Most of the recent papers on this topic are centered on certain fractional versions (time-fractional, space-fractional, space-time fractional) of the Poisson process, as well as some fractional birth processes (see, for instance, the review in Orsingher, 2013 and Alipour et al., 2015). Moreover, Beghin and Orsingher (2010) study the properties of Poisson-type fractional processes, governed by fractional recursive differential equations, obtained substituting regular derivatives with fractional derivatives. Mainardi et al. (2004) provide a generalization of the pure and compound Poisson processes via fractional calculus, by resorting to a renewal process-based approach involving waiting time distributions

Received by the editors August 23, 2015; accepted March 8, 2016.

2010 Mathematics Subject Classification. 60G22, 60J80, 60G40.

Key words and phrases. fractional difference-differential equations, Mittag-Leffler function, Wright function, random time, first passage time.

Research supported by GNCS-Indam and Regione Campania (Legge 5). 
expressed in term of the Mittag-Leffler function. A different approach has been developed by Laskin (2003) and Laskin (2009), where a fractional non-Markov Poisson stochastic process based on a fractional generalization of the Kolmogorov-Feller equations, and some interesting applications including a fractional compound Poisson process have been considered. More recently, Meerschaert et al. (2011) show that a Poisson process, with the time variable replaced by an independent inverse stable subordinator, is also a fractional Poisson process. Other recent results on fractional Poisson process can be found in Gorenflo and Mainardi (2012) and Gorenflo and Mainardi (2013).

Counting processes with jumps of amplitude larger than 1 are employed in various applications, since they are useful to describe simultaneous but independent Poisson streams (see Adelson (1966), for instance). The case of fractional compound Poisson processes has been investigated by Scalas (2012), Beghin and Macci (2012) and Beghin and Macci (2016+), for instance. Moreover, Beghin and Macci (2014) consider two fractional versions of nonnegative, integer-valued compound Poisson processes, and prove that their probability mass function solve certain fractional Kolmogorov forward equations. Certain fractional growth processes including the possibility of jumps of amplitude larger than 1 have been obtained recently through the interesting space-fractional Poisson process (cf. Orsingher and Polito (2012)) and, more generally, through the class of point processes studied in Orsingher and Toaldo (2015) and Polito and Scalas (2016). The relevance of fractional compound Poisson processes in applications in ruin theory and their long-range dependence are investigated in Biard and Saussereau (2014) and Maheshwari and Vellaisamy (2016+).

Following the lines of the papers above, here we analyse a suitable extension of the fractional Poisson process, say $M^{v}(t)$, which performs $k$ kinds of jumps of amplitude $1,2, \ldots, k$ with rates $\lambda_{1}, \lambda_{2}, \ldots, \lambda_{k}$ respectively. (Throughout the paper we refer to the fractional derivative in the Caputo sense, also known as Dzherbashyan-Caputo fractional derivative). We first obtain the moment generating function and the probability law of the process, and discuss its equivalent representation in terms of a subordinator governed by a suitable fractional Cauchy problem.

Along the same lines as Beghin and Orsingher (2010), in Section 2 we consider the difference-differential equations governing the probability mass function of $M^{v}(t)$ and involving the time-fractional derivative of order $v \in(0,1]$. The solution of the resulting Cauchy problem represents the probability distribution of the fractional counting process $M^{v}(t)$. Hence, we obtain $\mathbb{E}\left[e^{s M^{v}(t)}\right]$ and $p_{k}^{v}(t)=\mathbb{P}\left\{M^{v}(t)=k\right\}$ in terms of a generalized Mittag-Leffler function. We also show two useful representations for $M^{v}(t)$ :

(i) We prove that $M^{v}(t)$ can be expressed as a compound fractional Poisson process. This representation is essential to obtain a waiting time distribution.

(ii) We show that $M^{v}(t)$ can be regarded as a homogeneous Poisson process with $k$ kinds of jumps stopped at a random time. Such random time is the sole component of this subordinating relationship affected by the fractional derivative, since its distribution is obtained from the fundamental solution of a fractional diffusion equation.

In Section 3 we face the problem of determining certain waiting time and first-passagetime distributions. Specifically, we evaluate the probability that the first jump of size $j$, $j=1,2, \ldots, k$, for the process $M^{v}(t)$ occurs before time $t>0$. Interestingly, we prove that 
the first occurrence time of a jump of amplitude $j$ has the same distribution as the waiting time of the first event of the classical fractional Poisson process defined with parameter $\lambda_{j}$, for $j \in\{1,2, \ldots, k\}$. This is an immediate extension of a well-known result. Indeed, for a Poisson process with intensity $\lambda_{1}+\lambda_{2}$ and such that its events are classified as type $j$ via independent Bernoulli trials with probability $\frac{\lambda_{j}}{\lambda_{1}+\lambda_{2}}$, the first occurrence time of an event of type $j$ is distributed as the interarrival time of a Poisson process with intensity $\lambda_{j}$, $j=1,2$. In Theorem 3.1 we extend this result to the fractional setting. The remarkable difference is that the exponential density of the interarrival times of the Poisson process is replaced by the corresponding density of the fractional Poisson process, which depends on the (two-parameter) Mittal-Leffler function. In Section 3 we also study, when $k=2$, the distribution of the first passage time of $M^{v}(t)$ to a fixed level. We express it in an integral form which involves the joint distribution of the fractional Poisson process.

Finally, in Section 4 we obtain a formal expression of the moments of $M^{v}(t)$, and show that both the ratios given by the powers of the fractional Poisson process and of the process $M^{v}(t)$ over their means tend to 1 in probability. This result is useful in some applications. In fact, from a physical point of view, it means that the distance between the distributions of such processes at time $t$ and their equilibrium measures is close to 1 until some deterministic 'cutoff time' and is close to 0 shortly after.

In the remaining part of this section we briefly recall some well-known results on the fractional Poisson process which will be used throughout the paper. Consider the fractional Poisson process

$$
\left\{N_{\lambda}^{v}(t) ; t \geq 0\right\}, \quad v \in(0,1], \lambda \in(0, \infty),
$$

namely the renewal process with i.i.d. interarrival times $\mathscr{U}_{j}$ distributed according to the following density, for $j=1,2, \ldots$ and $t \in(0, \infty)$ (see Beghin and Orsingher, 2010):

$$
f_{1}^{v}(t)=\mathbb{P}\left\{\mathscr{U}_{j} \in d t\right\} / d t=\lambda t^{v-1} E_{v, v}\left(-\lambda t^{v}\right)
$$

where

$$
E_{\alpha, \beta}(x)=\sum_{r=0}^{\infty} \frac{x^{r}}{\Gamma(\alpha r+\beta)}, \quad \alpha, \beta \in \mathbb{C}, \operatorname{Re}(\alpha), \operatorname{Re}(\beta)>0, x \in \mathbb{R}
$$

is the (two-parameter) Mittag-Leffler function. From the Laplace transform

$$
\mathscr{L}\left\{f_{1}^{v}(t) ; s\right\}=\frac{\lambda}{s^{v}+\lambda}
$$

it follows that the density of the waiting time of the $k$-th event, $T_{k}=\sum_{j=1}^{k} \mathscr{U}_{j}$, possesses the Laplace transform

$$
\mathscr{L}\left\{f_{k}^{v}(t) ; s\right\}=\frac{\lambda^{k}}{\left(s^{v}+\lambda\right)^{k}}
$$

Its inverse can be obtained by applying formula (2.5) of Prabhakar (1971), i.e.

$$
\mathscr{L}\left\{{ }^{\gamma-1} E_{\beta, \gamma}^{\delta}\left(\omega t^{\beta}\right) ; s\right\}=\frac{s^{\beta \delta-\gamma}}{\left(s^{\beta}-\omega\right)^{\delta}},
$$

(where $\operatorname{Re}(\beta)>0, \operatorname{Re}(\gamma)>0, \operatorname{Re}(\delta)>0$ and $s>|\omega|^{\frac{1}{\operatorname{Re}(\beta)}}$ ). By setting $\beta=v, \gamma=k v, \delta=k$ and $\omega=-\lambda$ we have

$$
f_{k}^{v}(t)=\mathbb{P}\left\{T_{k} \in d t\right\} / d t=\lambda^{k} t^{k v-1} E_{v, k v}^{k}\left(-\lambda t^{v}\right),
$$


where

$$
E_{\alpha, \beta}^{\gamma}(z)=\sum_{r=0}^{\infty} \frac{(\gamma)_{r} z^{r}}{r ! \Gamma(\alpha r+\beta)}, \quad \alpha, \beta, \gamma \in \mathbb{C}, \operatorname{Re}(\alpha), \operatorname{Re}(\beta), \operatorname{Re}(\gamma)>0
$$

is a generalized Mittag-Leffler function and, as usual, $(\gamma)_{r}=\gamma(\gamma+1) \ldots(\gamma+r-1), r=$ $1,2, \ldots,(\gamma)_{0}=1$, is the Pochhammer symbol.

The corresponding distribution function can be obtained by integrating (1.4), thus obtaining (see Eq. (2.20) of Beghin and Orsingher, 2010)

$$
F_{k}^{v}(t)=\mathbb{P}\left\{T_{k}<t\right\}=\lambda^{k} t^{k v} E_{v, k v+1}^{k}\left(-\lambda t^{v}\right) .
$$

Taking into account (1.6), the probability mass function of the process $N_{\lambda}^{v}(t)$ can be easily computed as follows (see, also, Eq. (2.21) of Beghin and Orsingher, 2010):

$$
\mathbb{P}\left\{N_{\lambda}^{v}(t)=n\right\}=\mathbb{P}\left(T_{n} \leq t<T_{n+1}\right)=\left(\lambda t^{v}\right)^{n} E_{v, n v+1}^{n+1}\left(-\lambda t^{v}\right) .
$$

Moreover, recalling Eq. (2.29) of Beghin and Orsingher (2010), we have that the moment generating function of the process $N_{\lambda}^{v}(t), t \geq 0$, can be expressed as

$$
\mathbb{E}\left[e^{s N_{\lambda}^{v}(t)}\right]=E_{v, 1}\left(\lambda\left(e^{s}-1\right) t^{v}\right), \quad s \in \mathbb{R}
$$

The mean and the variance of $N_{\lambda}^{v}(t)$ read (see Eqs. (2.7) and (2.8) of Beghin and Orsingher, 2009)

$$
\mathbb{E}\left[N_{\lambda}^{v}(t)\right]=\frac{\lambda t^{v}}{\Gamma(v+1)}, \quad \operatorname{Var}\left[N_{\lambda}^{v}(t)\right]=\frac{2\left(\lambda t^{v}\right)^{2}}{\Gamma(2 v+1)}-\frac{\left(\lambda t^{v}\right)^{2}}{(\Gamma(v+1))^{2}}+\frac{\lambda t^{v}}{\Gamma(v+1)}
$$

In general, the analytical expression for the $m$ th order moment of the fractional Poisson process is given by (cf. Laskin (2009), Eq. (40))

$$
\mathbb{E}\left[\left(N_{\lambda}^{v}(t)\right)^{m}\right]=\sum_{l=0}^{m} S_{v}(m, l)\left(\lambda t^{v}\right)^{l}
$$

where $S_{v}(m, l)$ is the fractional Stirling number defined by Eq. (32) of Laskin (2009).

\section{Fractional counting process}

Let $\left\{M^{1}(t) ; t \geq 0\right\}$ be a counting process defined by following rules:

(1) $M^{1}(0)=0$ a.s.;

(2) $M^{1}(t)$ has stationary and independent increments;

(3) $\mathbb{P}\left\{M^{1}(h)=j\right\}=\lambda_{j} h+\mathrm{o}(h)$, for $j=1,2, \ldots, k$;

(4) $\mathbb{P}\left\{M^{1}(h)>k\right\}=\mathrm{o}(h)$,

where $k \in \mathbb{N} \equiv\{1,2, \ldots\}$ is fixed, and $\lambda_{1}, \lambda_{2}, \ldots, \lambda_{k}>0$. From the above assumptions we have that the probability distribution $p_{j}(t)=\mathbb{P}\left\{M^{1}(t)=j\right\}$, for $j \in \mathbb{N}_{0} \equiv\{0,1,2, \ldots\}$, satisfies the following system of difference-differential equations:

$$
\frac{\mathrm{d} p_{j}(t)}{\mathrm{d} t}=\sum_{r=1}^{k} \lambda_{r} p_{j-r}(t)-\left(\lambda_{1}+\ldots+\lambda_{k}\right) p_{j}(t), \quad t>0
$$

where $p_{j}(t)=0$ for $j<0$. 
In this section we examine a fractional extension of $\left\{M^{1}(t) ; t \geq 0\right\}$. We obtain a proper probability distribution and explore the main properties of the corresponding fractional process.

2.1. The probability law. With reference to the fractional derivatives

$$
\frac{\mathrm{d}^{v} f(t)}{\mathrm{d} t^{v}}= \begin{cases}\frac{1}{\Gamma(1-v)} \int_{0}^{t} \frac{(\mathrm{d} / \mathrm{d} s) f(s)}{(t-s)^{v}} \mathrm{~d} s, & 0<v<1 \\ f^{\prime}(t) & v=1\end{cases}
$$

let us now introduce a fractional extension of the process $M^{1}(t)$. For all fixed $v \in(0,1]$ and $k \in \mathbb{N}$, let $\left\{M^{v}(t) ; t \geq 0\right\}$ be a counting process, and assume that the probability distribution

$$
p_{j}^{v}(t)=\mathbb{P}\left\{M^{v}(t)=j\right\}, \quad j \in \mathbb{N}_{0}
$$

satisfies the following system of fractional difference-differential equations

$$
\left\{\begin{array}{l}
\frac{\mathrm{d} p_{0}^{v}(t)}{\mathrm{d} t^{v}}=-\Lambda p_{0}^{v}(t) \\
\frac{\mathrm{d} p_{j}^{v}(t)}{\mathrm{d} t^{v}}=\sum_{r=1}^{j} \lambda_{r} p_{j-r}^{v}(t)-\Lambda p_{j}^{v}(t), \quad j=1,2, \ldots, k-1 \\
\frac{\mathrm{d} p_{j}^{v}(t)}{\mathrm{d} t^{v}}=\sum_{r=1}^{k} \lambda_{r} p_{j-r}^{v}(t)-\Lambda p_{j}^{v}(t), \quad j=k, k+1, \ldots,
\end{array}\right.
$$

for $\Lambda=\lambda_{1}+\lambda_{2}+\ldots+\lambda_{k}$, together with the condition

$$
p_{j}(0)= \begin{cases}1, & j=0 \\ 0, & j \geq 1\end{cases}
$$

Clearly, when $v=1$ the system (2.3) identifies with the difference-differential equations of process $M^{1}(t)$ given in (2.1). Furthermore, when $k=1$ the process $M^{v}(t)$ identifies with the process $N_{\lambda}^{v}(t)$ considered in Section 1.

Hereafter we will obtain the solution to (2.3)-(2.4) in terms of the generalized MittagLeffler function (1.5) and show that it represents a true probability distribution of $M^{v}(t)$. To this purpose we first obtain the moment generating function of $M^{v}(t)$ in terms of the Mittag-Leffler function.

Proposition 2.1. For all fixed $v \in(0,1]$ and $k \in \mathbb{N}$, the moment generating function of $M^{v}(t)$ is given by

$$
\mathbb{E}\left[e^{s M^{v}(t)}\right]=E_{v, 1}\left(\sum_{j=1}^{k} \lambda_{j}\left(e^{j s}-1\right) t^{v}\right), \quad t \geq 0, s \in \mathbb{R} .
$$

Proof: From system (2.3) and condition (2.4) we have that the probability generating function $G(z, t):=\mathbb{E}\left[z^{M^{v}(t)}\right]$ satisfies the Cauchy problem

$$
\left\{\begin{array}{l}
\frac{\partial G(z, t)}{\partial t^{v}}=-\sum_{j=1}^{k} \lambda_{j}\left(1-z^{j}\right) G(z, t) \\
G(z, 0)=1 .
\end{array}\right.
$$

By adopting a Laplace-transform approach we obtain

$$
\mathscr{L}\{G(z, t) ; s\}=\frac{s^{v-1}}{s^{v}+\sum_{j=1}^{k} \lambda_{j}\left(1-z^{j}\right)} .
$$


Eq. (2.5) thus follows recalling Eq. (1.3).

We remark that the use of the Caputo fractional derivative permits us to avoid fractional initial conditions in the previous proof since, in general,

$$
\mathscr{L}\left\{f^{v} ; s\right\}=s^{v} \mathscr{L}\{f ; s\}-\left.s^{v-1} f\right|_{x=0}, \quad v \in(0,1] .
$$

Let us now show that $M^{v}(t)$ can be expressed as a compound fractional Poisson process.

Proposition 2.2. For all fixed $v \in(0,1]$ we have

$$
M^{v}(t) \stackrel{d}{=} \sum_{i=1}^{N_{\Lambda}^{v}(t)} X_{i}, \quad t \geq 0,
$$

where $N_{\Lambda}^{v}(t)$ is a fractional Poisson process, defined as in (1.1), with intensity $\Lambda=\lambda_{1}+$ $\lambda_{2}+\ldots+\lambda_{k}$. Moreover, $\left\{X_{n}: n \geq 1\right\}$ is a sequence of i.i.d. random variables, independent of $N_{\Lambda}^{V}(t)$, such that for any $n \in \mathbb{N}$

$$
\mathbb{P}\left\{X_{n}=j\right\}=\frac{\lambda_{j}}{\Lambda}, \quad j=1,2, \ldots, k
$$

and where both $N_{\Lambda}^{v}(t)$ and $X_{n}$ depend on the same parameters $\lambda_{1}, \lambda_{2}, \ldots, \lambda_{k}$.

Proof: The moment generating function of $Y(t):=\sum_{i=1}^{N_{\Lambda}^{v}(t)} X_{i}, t \geq 0$, can be expressed as

$$
\begin{aligned}
\mathbb{E}\left[e^{s Y(t)}\right] & =\mathbb{E}\left[\mathbb{E}\left[e^{s Y(t)} \mid N_{\Lambda}^{v}(t)\right]\right] \\
& =\mathbb{E}\left[\left(\mathbb{E}\left[e^{s X_{1}}\right]\right)^{N_{\Lambda}^{v}(t)}\right] .
\end{aligned}
$$

Hence, since

$$
\mathbb{E}\left[e^{s X_{1}}\right]=\frac{1}{\Lambda} \sum_{j=1}^{k} \lambda_{j} e^{j s}
$$

we have

$$
\mathbb{E}\left[e^{s Y(t)}\right]=\mathbb{E}\left[e^{N_{\Lambda}^{v}(t) \ln \left(\frac{1}{\Lambda} \sum_{j=1}^{k} \lambda_{j} e^{j s}\right)}\right] .
$$

Finally, making use of Eq. (1.8) we immediately obtain that the moment generating function of $Y(t)$ identifies with the right-hand-side of (2.5). This completes the proof.

We remark that, due to Proposition 2.2, $M^{v}(t)$ can be regarded as a special case of the process defined in Eq. (7) of Beghin and Macci (2014), under a suitable choice of the probability mass function $\left(q_{k}\right)_{k \geq 1}$ and the parameter $\lambda$. Furthermore, according to Definition 7.1.1 of Bening and Korolev (2002), the process $M^{v}(t)$ is a compound Cox process, since Beghin and Orsingher (2010) show that $N_{\Lambda}^{V}(t)$ is a Cox process with a proper directing measure. Moreover, $M^{v}(t)$ is a compound fractional process, and thus it is neither Markovian nor Lèvy (cf. Scalas, 2012).

We are now able to obtain the probability mass function (2.2) of $M^{v}(t)$. Indeed, the following Proposition holds true.

Proposition 2.3. The solution $p_{j}^{v}(t)$ of the Cauchy problem (2.3)-(2.4), for $j \in \mathbb{N}_{0}, v \in$ $(0,1]$ and $t \geq 0$, is given by

$$
p_{j}^{v}(t)=\sum_{r=0}^{j} \sum_{\begin{array}{c}
\alpha_{1}+\alpha_{2}+\ldots+\alpha_{k}=r \\
\alpha_{1}+2 \alpha_{2}+\ldots+k \alpha_{k}=j
\end{array}}\left(\begin{array}{c}
r \\
\alpha_{1}, \alpha_{2}, \ldots, \alpha_{k}
\end{array}\right) \lambda_{1}^{\alpha_{1}} \lambda_{2}^{\alpha_{2}} \ldots \lambda_{k}^{\alpha_{k}} t^{r v} E_{v, r v+1}^{r+1}\left(-\Lambda t^{v}\right) .
$$


FIGURE 2.1. Probability distribution of $M^{v}(t)$, given in (2.8), for $j=$ $0,1, \ldots, 11$, with $k=3, v=0.5, \lambda_{1}=\lambda_{2}=\lambda_{3}=1,(a) t=1$ and $(b)$ $t=2$. The displayed probability mass is $(a) 0.797292$ and $(b) 0.629278$.

Proof: From (2.6) and from a conditioning argument we have

$$
p_{j}^{v}(t)=\mathbb{P}\left\{M^{v}(t)=j\right\}=\sum_{r=0}^{j} \mathbb{P}\left\{X_{1}+X_{2}+\ldots+X_{r}=j\right\} \mathbb{P}\left\{N_{\Lambda}^{v}(t)=r\right\} .
$$

Since $X_{1}, X_{2}, \ldots, X_{r}$ are independent and identically distributed (cf. (2.7)), it follows that

$$
\begin{aligned}
\mathbb{P}\left\{X_{1}+X_{2}+\ldots+X_{r}=j\right\}= & \sum_{\substack{\alpha_{1}+\alpha_{2}+\ldots+\alpha_{k}=r \\
\alpha_{1}+2 \alpha_{2}+\ldots+k \alpha_{k}=j}}\left(\begin{array}{c}
r \\
\alpha_{1}, \alpha_{2}, \ldots, \alpha_{k}
\end{array}\right) \\
& \times\left(\frac{\lambda_{1}}{\Lambda}\right)^{\alpha_{1}}\left(\frac{\lambda_{2}}{\Lambda}\right)^{\alpha_{2}} \ldots\left(\frac{\lambda_{k}}{\Lambda}\right)^{\alpha_{k}},
\end{aligned}
$$

where the sum is taken in order to consider all the possible ways of having $r$ jumps, with $\alpha_{1}$ jumps of size $1, \ldots, \alpha_{k}$ jumps of size $k$, and such that the total amplitude, i.e. $\alpha_{1}+$ $2 \alpha_{2}+\ldots+k \alpha_{k}$, equals $j$. Hence, recalling formula (1.7), the Proposition follows.

Proposition 2.3 is an extension of Proposition 2 of Di Crescenzo et al. (2015), which is concerning with case $k=2$. Some plots of probabilities (2.8) are shown in Figure 2.1 and Figure 2.2.

From (2.8) we note that, for $v \in(0,1]$,

$$
p_{0}^{v}(t)=E_{v, 1}\left(-\Lambda t^{v}\right), \quad t \geq 0 .
$$

Moreover, making use of Eqs. (1.5) and (2.8) we obtain hereafter the distribution of the process $M^{v}(t)$ in the special case $v=1$.

Corollary 2.4. The probability mass function $p_{j}^{1}(t)$, for $j \in \mathbb{N}_{0}$ and $t \geq 0$, is given by

$$
p_{j}^{1}(t)=\sum_{r=0}^{j} \sum_{\substack{\alpha_{1}+\alpha_{2}+\ldots+\alpha_{k}=r \\ \alpha_{1}+2 \alpha_{2}+\ldots+k \alpha_{k}=j}} \frac{\lambda_{1}^{\alpha_{1}} \lambda_{2}^{\alpha_{2}} \ldots \lambda_{k}^{\alpha_{k}}}{\alpha_{1} ! \alpha_{2} ! \ldots \alpha_{k} !} t^{r} e^{-\Lambda t}
$$

2.2. Equivalent representation. We will now examine an interesting relationship between the process $M^{v}(t)$ and the process $M^{1}(t)$. In fact, we show that the following representation holds:

$$
M^{v}(t) \stackrel{d}{=} M^{1}\left(\mathscr{T}_{2 v}(t)\right)
$$


FIGURE 2.2. Probability distribution of $M^{v}(t)$, given in (2.8), for $0 \leq$ $t \leq 2$, with $k=3, \lambda_{1}=\lambda_{2}=\lambda_{3}=1,(a) v=0.5$ and $(b) v=1$.

where $\mathscr{T}_{2 v}(t)$ is a suitable random process, and thus $M^{v}(t)$ can be considered as a homogeneous Poisson-type counting process with jumps of sizes $1,2, \ldots, k$ stopped at a random time $\mathscr{T}_{2 v}(t)$.

Let us denote by $g(z, t)=g_{2 v}(z, t)$ the solution of the Cauchy problem

$$
\begin{cases}\frac{\partial^{2 v} g(z, t)}{\partial t^{2 v}}=\frac{\partial^{2} g(z, t)}{\partial z^{2}}, & t>0, z \in \mathbb{R} \\ g(z, 0)=\delta(z), & 0<v<1 \\ \left.\frac{\partial g(z, t)}{\partial t}\right|_{t=0}=0, & \frac{1}{2}<v<1 .\end{cases}
$$

It is well-known that (see Mainardi, 1996b and Mainardi, 1996a)

$$
g_{2 v}(z, t)=\frac{1}{2 t^{v}} W_{-v, 1-v}\left(-\frac{|z|}{t^{v}}\right), \quad t>0, z \in \mathbb{R},
$$

where

$$
W_{\alpha, \beta}(x)=\sum_{k=0}^{\infty} \frac{x^{k}}{k ! \Gamma(\alpha k+\beta)}, \quad \alpha>-1, \beta>0, x \in \mathbb{R}
$$

is the Wright function. Let

$$
\bar{g}_{2 v}(z, t)= \begin{cases}2 g_{2 v}(z, t), & z>0 \\ 0, & z<0\end{cases}
$$

be the folded solution to (2.10) and let $\mathscr{T}_{2 v}(t)$ be a random process (independent from the process $\left.M^{1}(t)\right)$ whose transition density $\mathbb{P}\left\{\mathscr{T}_{2 v}(t) \in d z\right\} / d z$ is given in (2.13).

Remark 2.5. It has been proved in Orsingher and Beghin (2004) that the solution $g_{2 v}$ to (2.10) can be alternatively expressed as

$$
g_{2 v}(z, t)=\frac{1}{2 \Gamma(1-v)} \int_{0}^{t}(t-w)^{-v} f_{v}(w,|z|) d w, \quad z \in \mathbb{R},
$$

where $f_{v}(\cdot, y)$ is a stable law $S_{v}(\mu, \beta, \sigma)$ of order $v$, with parameters $\mu=0, \beta=1$ and $\sigma=\left(z \cos \frac{\pi v}{2}\right)^{\frac{1}{v}}$.

Proposition 2.6. The process $M^{v}(t)$ and the process $M^{1}\left(\mathscr{T}_{2 v}(t)\right)$ are identically distributed. 
Proof: From (2.2) and (2.13) we have

$$
\mathbb{P}\left\{M^{1}\left(\mathscr{T}_{2 v}(t)\right)=n\right\}=\int_{0}^{\infty} p_{n}^{1}(z) \bar{g}_{2 v}(z, t) d z .
$$

Hence, making use of (2.9) and (2.12) we get

$$
\begin{aligned}
\mathbb{P}\left\{M^{1}\left(\mathscr{T}_{2 v}(t)\right)=n\right\}= & \sum_{j=0}^{n} \sum_{\substack{\alpha_{1}+\alpha_{2}+\ldots+\alpha_{k}=j \\
\alpha_{1}+2 \alpha_{2}+\ldots+k \alpha_{k}=n}} \frac{\lambda_{1}^{\alpha_{1}} \lambda_{2}^{\alpha_{2}} \ldots \lambda_{k}^{\alpha_{k}}}{\alpha_{1} ! \alpha_{2} ! \ldots \alpha_{k} !} \\
& \times \frac{1}{t^{v}} \int_{0}^{\infty} e^{-\Lambda z} z^{j} W_{-v, 1-v}\left(-\frac{z}{t^{v}}\right) d z .
\end{aligned}
$$

For $y=\Lambda z$, the last expression identifies with (2.8) due to the following integral representation of the generalized Mittag-Leffler function in terms of the Wright function, proposed by Beghin and Orsingher (2010):

$$
E_{v, k v+1}^{k+1}\left(-\Lambda t^{v}\right)=\frac{1}{k ! \Lambda^{k+1} t^{(k+1) v}} \int_{0}^{\infty} e^{-y} y^{k} W_{-v, 1-v}\left(-\frac{y}{\Lambda t^{v}}\right) d y .
$$

This completes the proof.

Remark 2.7. Since the transition density (2.13) coincides with the probability density function of the standard inverse $v$-stable subordinator $\mathrm{E}^{v}(t)$ (see Meerschaert et al., 2011), the result given in Proposition 2.6 can be stated also as follows: The process $M^{v}(t)$ and the process $M^{1}\left(\mathrm{E}^{v}(t)\right)$ are identically distributed.

Remark 2.8. In Beghin and Orsingher (2010) Beghin and Orsingher proved an analogous subordination relationship, i.e.

$$
N_{\lambda}^{v}(t) \stackrel{d}{=} N_{\lambda}^{1}\left(\mathscr{T}_{2 v}(t)\right)
$$

where $N_{\lambda}^{v}(t)$ is the fractional Poisson process defined in (1.1) and $\mathscr{T}_{2 v}(t)$ is the random time defined above.

Remark 2.9. By taking $v=\frac{1}{2}$, from Proposition 2.6 we have that $M^{1 / 2}(t)$ and $M^{1}\left(\mathscr{T}_{1}(t)\right)$ are identically distributed. We note that the random time $\mathscr{T}_{1}(t), t>0$, becomes a reflecting Brownian motion. Indeed, in this case equation (2.10) reduces to the heat equation

$$
\left\{\begin{array}{l}
\frac{\partial g}{\partial t}=\frac{\partial^{2} g}{\partial z^{2}}, \quad t>0, z \in \mathbb{R} \\
g(z, 0)=\delta(z),
\end{array}\right.
$$

and the solution $g_{1}(z, t)$ is the density of a Brownian motion $B(t), t>0$, with infinitesimal variance 2 . After folding up the solution, we find the following probability mass

$$
\begin{aligned}
\mathbb{P}\left\{M^{1}\left(\mathscr{T}_{1}(t)\right)=n\right\} & =\int_{0}^{\infty} p_{n}^{1}(z) \frac{e^{-\frac{z^{2}}{4 t}}}{\sqrt{\pi} t} d z \\
& =\mathbb{P}\left\{M^{1}(|B(t)|)=n\right\},
\end{aligned}
$$

so that $M^{1 / 2}(t)$ is a jump process at a Brownian time.

Remark 2.10. It is worth noticing that both the compositions of the fractional Poisson process $N_{\lambda}^{v}(t)$ defined in (1.1) and of the fractional process $M^{v}(t)$ defined in (2.2) with the random time $\mathscr{T}_{2 v}(t)$ yields again fractional processes of different order, i.e.

$$
N_{\lambda}^{v}\left(\mathscr{T}_{2 v}(t)\right) \stackrel{d}{=} N_{\lambda}^{v^{2}}(t) \quad \text { and } \quad M^{v}\left(\mathscr{T}_{2 v}(t)\right) \stackrel{d}{=} M^{v^{2}}(t) .
$$


Taking into account the subordinating relationships examined in Proposition 2.6 and in Remark 2.8, this fact follows immediately from Remark 3.1 of Kumar et al. (2011), since, in general, the composition of two stable subordinators of indexes $\beta_{1}$ and $\beta_{2}$ respectively is a stable subordinator of index $\beta_{1} \beta_{2}$.

Remark 2.11. Bearing in mind Proposition 2.2, setting

$$
\mathscr{S}_{r}=\Lambda \cdot \mathbb{E}\left[X^{r}\right]=\sum_{j=1}^{k} j^{r} \lambda_{j}, \quad r=1,2
$$

and recalling (1.9), we can compute more effortlessly the mean and the variance of the process. In fact, by Wald's equation we have

$$
\begin{aligned}
\mathbb{E}\left[M^{v}(t)\right] & =\mathbb{E}[X] \cdot \mathbb{E}\left[N_{\Lambda}^{v}(t)\right] \\
& =\frac{\mathscr{S}_{1} t^{v}}{\Gamma(v+1)}, \quad t \geq 0 .
\end{aligned}
$$

Moreover, by the law of total variance we get

$$
\begin{aligned}
\operatorname{Var}\left[M^{v}(t)\right] & =\operatorname{Var}[X] \cdot \mathbb{E}\left[N_{\Lambda}^{v}(t)\right]+(\mathbb{E}[X])^{2} \cdot \operatorname{Var}\left[N_{\Lambda}^{v}(t)\right] \\
& =\frac{\mathscr{S}_{2} t^{v}}{\Gamma(v+1)}+\mathscr{S}_{1}^{2} t^{2 v} Z(v), \quad t \geq 0
\end{aligned}
$$

where

$$
Z(v):=\frac{1}{v}\left(\frac{1}{\Gamma(2 v)}-\frac{1}{v \Gamma^{2}(v)}\right)
$$

As a consequence it is not hard to show that $\operatorname{Var}\left[M^{v}(t)\right]-\mathbb{E}\left[M^{v}(t)\right]>0$, or, equivalently, that the process $M^{v}(t)$ exhibits overdispersion, since $Z(v)>0$ for all $v \in(0,1)$ and $Z(1)=$ 0 . Finally, we point out that a formal expression for the moments of process $M^{v}(t)$ is provided in Lemma 4.1.

\section{Waiting times and first-passage times}

We evaluate the probability distribution function of the waiting time until the first occurrence of a jump of size $i, i=1,2, \ldots, k$, for the process $M^{v}(t)$. We first observe that the following decomposition holds:

$$
M^{v}(t)=\sum_{j=1}^{k} j M_{j}^{v}(t), \quad t \geq 0
$$

where

$$
M_{j}^{v}(t):=\sum_{i=1}^{N_{\Lambda}^{v}(t)} \mathbf{1}_{\left\{X_{i}=j\right\}}, \quad j=1,2, \ldots, k,
$$

and thus $M_{j}^{v}(t)$ counts the number of jumps of amplitude $j$ performed by $M^{v}(t)$ in $(0, t]$. Furthermore, we introduce the random variables

$$
H_{j}:=\inf \left\{s>0: M_{j}^{v}(s)=1\right\} \quad \text { and } \quad G^{j} \sim \operatorname{Geo}\left(\frac{\lambda_{j}}{\Lambda}\right), \quad j=1,2, \ldots, k
$$

In other words, $H_{j}$ represents the first occurrence time of a jump of amplitude $j$ for process $M^{v}(t)$, whereas $G^{j}$ is a geometric random variable with parameter $\frac{\lambda_{j}}{\Lambda}$ that describes the order of the first jump of amplitude $j$ in the sequence of jumps of $M^{v}(t)$. We prove that $H_{j}$ is distributed as the waiting time of the first event of the fractional Poisson process defined in (1.1) with parameter $\lambda_{j}$. Indeed, the following result holds. 
Theorem 3.1. Let $j \in\{1,2, \ldots, k\}$. Then

$$
\mathbb{P}\left\{H_{j} \leq t\right\}=\lambda_{j} t^{v} E_{v, v+1}\left(-\lambda_{j} t^{v}\right), \quad t>0 .
$$

Proof: By conditioning on $G^{j}$, for $t>0$, due to Eqs. (2.6) and (1.6) we have

$$
\begin{aligned}
\mathbb{P}\left\{H_{j} \leq t\right\} & =\mathbb{E}_{G^{j}}\left[\mathbb{P}\left\{H_{j} \leq t \mid G^{j}\right\}\right] \\
& =\sum_{n=1}^{+\infty} \mathbb{P}\left\{H_{j} \leq t \mid G^{j}=n\right\} \mathbb{P}\left\{G^{j}=n\right\} \\
& =\sum_{n=1}^{+\infty} F_{n}^{v}(t) \frac{\lambda_{j}}{\Lambda}\left(1-\frac{\lambda_{j}}{\Lambda}\right)^{n-1} \\
& =\sum_{n=1}^{+\infty} \Lambda^{n} t^{n v} E_{v, n v+1}^{n}\left(-\Lambda t^{v}\right) \frac{\lambda_{j}}{\Lambda}\left(1-\frac{\lambda_{j}}{\Lambda}\right)^{n-1} \\
& =\lambda_{j} t^{v} \sum_{n=0}^{+\infty} \Lambda^{n} t^{n v}\left(1-\frac{\lambda_{j}}{\Lambda}\right)^{n} E_{v,(n+1) v+1}^{n+1}\left(-\Lambda t^{v}\right) .
\end{aligned}
$$

By using formula (2.3.1) of Mathai and Haubold (2008), i.e.

$$
\frac{1}{\Gamma(\alpha)} \int_{0}^{1} u^{\gamma-1}(1-u)^{\alpha-1} E_{\beta, \gamma}^{\delta}\left(z u^{\beta}\right) d u=E_{\beta, \gamma+\alpha}^{\delta}(z)
$$

(where $\operatorname{Re}(\alpha)>0, \operatorname{Re}(\beta)>0$ and $\operatorname{Re}(\gamma)>0$ ) for $\alpha=\beta=v, \gamma=n v+1, \delta=n+1$ and $z=-\Lambda t^{v}$, we get

$$
\begin{aligned}
\mathbb{P}\left\{H_{j} \leq t\right\} & =\frac{\lambda_{j} t^{v}}{\Gamma(v)} \sum_{n=0}^{+\infty} \Lambda^{n} t^{n v}\left(1-\frac{\lambda_{j}}{\Lambda}\right)^{n} \int_{0}^{1} u^{n v}(1-u)^{v-1} E_{v, n v+1}^{n+1}\left(-\Lambda t^{v} u^{v}\right) d u \\
& =\frac{\lambda_{j} t^{v}}{\Gamma(v)} \int_{0}^{1}(1-u)^{v-1} \sum_{n=0}^{+\infty}\left[\Lambda t^{v}\left(1-\frac{\lambda_{j}}{\Lambda}\right) u^{v}\right]^{n} E_{v, n v+1}^{n+1}\left(-\Lambda t^{v} u^{v}\right) d u
\end{aligned}
$$

Due to formula (2.30) of Beghin and Orsingher (2010), i.e.

$$
\sum_{n=0}^{+\infty}\left(\lambda w t^{v}\right)^{n} E_{v, v n+1}^{n+1}\left(-\lambda t^{v}\right)=E_{v, 1}\left(\lambda(w-1) t^{v}\right) \quad(|w| \leq 1, t>0),
$$

we have

$$
\mathbb{P}\left\{H_{j} \leq t\right\}=\frac{\lambda_{j} t^{v}}{\Gamma(v)} \int_{0}^{1}(1-u)^{v-1} E_{v, 1}\left(-\lambda_{j} t^{v} u^{v}\right) d u
$$

By making use of formula (2.2.14) of Mathai and Haubold (2008), i.e.

$$
\int_{0}^{1} z^{\beta-1}(1-z)^{\sigma-1} E_{\alpha, \beta}\left(x z^{\alpha}\right) d z=\Gamma(\sigma) E_{\alpha, \sigma+\beta}(x),
$$

(where $\alpha>0 ; \beta, \sigma \in \mathbb{C} ; \operatorname{Re}(\beta)>0$ and $\operatorname{Re}(\sigma)>0$ ), for $\sigma=\alpha=v, \beta=1$ and $x=-\lambda_{j} t^{v}$, we get

$$
\mathbb{P}\left\{H_{j} \leq t\right\}=\lambda_{j} t^{v} E_{v, v+1}\left(-\lambda_{j} t^{v}\right), \quad t \geq 0 .
$$

Therefore $H_{j}$ is distributed as the waiting time of the first event of the fractional Poisson process defined in (1.1) (cf. (1.6)).

The result shown in Theorem 3.1 is an immediate extension of the well-known result for the Poisson process, i.e. for $v=1$, by which $H_{j}$ is exponentially distributed with parameter $\lambda_{j}$. 
We will now be concerned with the distribution of the first passage time to a fixed level for the process $M^{v}(t)$, denoted as

$$
\tau_{n}=\inf \left\{s>0: M^{v}(s)=n\right\}, \quad n \in \mathbb{N} .
$$

The following result is concerning the case $k=2$, i.e. when the process $M^{v}(t)$ performs jumps of sizes 1 and 2 .

Theorem 3.2. The cumulative distribution function of the first passage time $\tau_{k}$ when $k=2$ reads

$$
\begin{aligned}
\mathbb{P}\left\{\tau_{n} \leq t\right\}= & \sum_{h=n}^{+\infty} \sum_{j=\left\lceil\frac{h}{2}\right\rceil}^{h} \sum_{i=1}^{j}\left(\begin{array}{c}
i \\
n-i
\end{array}\right)\left(\begin{array}{c}
j-i \\
h-n-j+i
\end{array}\right)\left(\frac{\lambda_{1}}{\lambda_{1}+\lambda_{2}}\right)^{2 j-h}\left(\frac{\lambda_{2}}{\lambda_{1}+\lambda_{2}}\right)^{h-j} \\
& \times \int_{0}^{t} \mathbb{P}\left\{N_{\lambda_{1}+\lambda_{2}}^{v}(t)=j, N_{\lambda_{1}+\lambda_{2}}^{v}(s)=i\right\} d s, \quad t>0 .
\end{aligned}
$$

Proof: Since the process $M^{v}(t)$ performs jumps of size 1 and 2, and has non-independent increments, the computation of the cumulative distribution function of the first passage time (3.3) can be carried out as follows:

$$
\begin{aligned}
\mathbb{P}\left\{\tau_{n} \leq t\right\}= & \sum_{h=n}^{+\infty} \int_{0}^{t} \mathbb{P}\left\{M^{v}(t)=h, M^{v}(s)=n\right\} d s \\
= & \sum_{h=n}^{+\infty} \sum_{j=\left\lceil\frac{h}{2}\right\rceil}^{h} \sum_{i=1}^{j} \int_{0}^{t} \mathbb{P}\left\{M^{v}(t)=h, M^{v}(s)=n \mid N_{\lambda_{1}+\lambda_{2}}^{v}(t)=j, N_{\lambda_{1}+\lambda_{2}}^{v}(s)=i\right\} \\
& \times \mathbb{P}\left\{N_{\lambda_{1}+\lambda_{2}}^{v}(t)=j, N_{\lambda_{1}+\lambda_{2}}^{v}(s)=i\right\} d s .
\end{aligned}
$$

Making use of Proposition 2.2 we have:

$$
\begin{aligned}
\mathbb{P}\left\{\tau_{n} \leq t\right\}= & \sum_{h=n}^{+\infty} \sum_{j=\left\lceil\frac{h}{2}\right\rceil}^{h} \sum_{i=1}^{j} \int_{0}^{t} \mathbb{P}\left\{\sum_{r=1}^{j} X_{r}=h, \sum_{l=1}^{i} X_{l}=n\right\} \\
& \times \mathbb{P}\left\{N_{\lambda_{1}+\lambda_{2}}^{v}(t)=j, N_{\lambda_{1}+\lambda_{2}}^{v}(s)=i\right\} d s \\
= & \sum_{h=n}^{+\infty} \sum_{j=\left\lceil\frac{h}{2}\right\rceil}^{h} \sum_{i=1}^{j} \int_{0}^{t} \mathbb{P}\left\{\sum_{l=1}^{i} X_{l}=n, \sum_{r=i+1}^{j} X_{r}=h-n\right\} \\
& \times \mathbb{P}\left\{N_{\lambda_{1}+\lambda_{2}}^{v}(t)=j, N_{\lambda_{1}+\lambda_{2}}^{v}(s)=i\right\} d s \\
= & \sum_{h=n}^{+\infty} \sum_{j=\left\lceil\frac{h}{2}\right\rceil}^{h} \sum_{i=1}^{j} \int_{0}^{t} \mathbb{P}\left\{\sum_{l=1}^{i} X_{l}=n\right\} \mathbb{P}\left\{\sum_{r=i+1}^{j} X_{r}=h-n\right\} \\
& \times \mathbb{P}\left\{N_{\lambda_{1}+\lambda_{2}}^{v}(t)=j, N_{\lambda_{1}+\lambda_{2}}^{v}(s)=i\right\} d s \\
= & \sum_{h=n}^{+\infty} \sum_{j=\left\lceil\frac{h}{2}\right\rceil}^{h} \sum_{i=1}^{j}\left(\begin{array}{c}
i \\
n-i
\end{array}\right)\left(\frac{\lambda_{1}}{\lambda_{1}+\lambda_{2}}\right)^{2 i-n}\left(\frac{\lambda_{2}}{\lambda_{1}+\lambda_{2}}\right)^{n-i} \\
& \times\left(\begin{array}{c}
j-i \\
h-n-j+i
\end{array}\right)\left(\frac{\lambda_{1}}{\lambda_{1}+\lambda_{2}}\right)^{2 j-2 i+n-h}\left(\frac{\lambda_{2}}{\lambda_{1}+\lambda_{2}}\right)^{h-n-j+i} \\
& \times \int_{0}^{t} \mathbb{P}\left\{N_{\lambda_{1}+\lambda_{2}}^{v}(t)=j, N_{\lambda_{1}+\lambda_{2}}^{v}(s)=i\right\} d s,
\end{aligned}
$$


this giving Eq. (3.4).

To the best of our knowledge, the bivariate distribution shown in the right-hand-side of (3.4), i.e. $\mathbb{P}\left\{N_{\lambda_{1}+\lambda_{2}}^{v}(s)=i, N_{\lambda_{1}+\lambda_{2}}^{v}(t)=j\right\}$, cannot be expressed in a closed form. Orsingher and Polito (2013) derived an expression in terms of Prabhakar integrals, i.e.:

$$
\begin{aligned}
\mathbb{P}\left\{N_{\lambda_{1}+\lambda_{2}}^{v}(s)=i, N_{\lambda_{1}+\lambda_{2}}^{v}(t)=j\right\} & \\
=\left(\lambda_{1}+\lambda_{2}\right)^{j}\left(\mathbf{E}_{v, v i,-\left(\lambda_{1}+\lambda_{2}\right) ;(t-s)+}^{i}\right. & \mathbf{E}_{v, v(j-i-1)+1,-\left(\lambda_{1}+\lambda_{2}\right) ;(z+s-t)+}^{j-i} \\
& \left.\left.\times y^{v-1} E_{v, v}\left(-\left(\lambda_{1}+\lambda_{2}\right) y^{v}\right)\right)(z)\right)(t),
\end{aligned}
$$

where

$$
\left(\mathbf{E}_{\rho, \mu, \omega ; a+}^{\gamma} \phi\right)(x)=\int_{a}^{x}(x-t)^{\mu-1} E_{\rho, \mu}^{\gamma}\left(\omega(x-t)^{\rho}\right) \phi(t) d t
$$

is the Prabhakar integral (see Prabhakar (1971) for details). Politi et al. (2011), instead, evaluate the joint probability given in (3.4) by introducing the random variable $Y_{i}$ which denotes the residual lifetime at $s$ (that is the time to the next epoch) conditional on $N_{\lambda_{1}+\lambda_{2}}^{v}(s)=$ $i$, i.e. $Y_{i} \stackrel{\text { def }}{=}\left[\tau_{i}-s \mid N_{\lambda_{1}+\lambda_{2}}^{v}(s)=i\right]$ whose cumulative distribution function is denoted by $F_{Y_{i}}(y)$. Therefore,

$$
\begin{aligned}
\mathbb{P}\left\{N_{\lambda_{1}+\lambda_{2}}^{v}(s)=i, N_{\lambda_{1}+\lambda_{2}}^{v}(t)=j\right\}= & \mathbb{P}\left\{N_{\lambda_{1}+\lambda_{2}}^{v}(t)-N_{\lambda_{1}+\lambda_{2}}^{v}(s)=j-i \mid N_{\lambda_{1}+\lambda_{2}}^{v}(s)=i\right\} \\
& \times \mathbb{P}\left\{N_{\lambda_{1}+\lambda_{2}}^{v}(s)=i\right\},
\end{aligned}
$$

where

$$
\begin{aligned}
\mathbb{P}\left\{N_{\lambda_{1}+\lambda_{2}}^{v}(t)-N_{\lambda_{1}+\lambda_{2}}^{v}(s)=j-i \mid N_{\lambda_{1}+\lambda_{2}}^{v}(s)=i\right\} & \\
= & \begin{cases}\int_{0}^{t-s} \mathbb{P}\left\{N_{\lambda_{1}+\lambda_{2}}^{v}(t-s-y)=j-i-1\right\} d F_{Y_{i}}(y), & \text { if } j-i \geq 1, \\
1-F_{Y_{i}}(t-s), & \text { if } j-i=0 .\end{cases}
\end{aligned}
$$

It is meaningful to stress that when $k=2$ the passage of $M^{v}(t)$ to a level $n$ is not sure. In fact, the process can cross state $n$ without visiting it due to the effect of a jump having size 2.

\section{Convergence results}

For the processes $N_{\lambda}^{v}(t)$ and $M^{v}(t)$, defined respectively in (1.1) and in (2.2), we now focus on a property related to their asymptotic behavior as the relevant parameters grow larger.

Proposition 4.1. Let $v \in(0,1]$. Then for a fixed $t>0$ we have

$$
\frac{N_{\lambda}^{v}(t)}{\mathbb{E}\left[N_{\lambda}^{v}(t)\right]} \underset{\lambda \rightarrow+\infty}{\stackrel{\text { Prob }}{\longrightarrow}} 1 \text {. }
$$


Proof: We study the convergence in mean of the random variable $\frac{N_{\lambda}^{v}(t)}{\mathbb{E}\left[N_{\lambda}^{v}(t)\right]}$ to 1 . Due to the triangular inequality we have

$$
\mathbb{E}\left[\left|\frac{N_{\lambda}^{v}(t)}{\mathbb{E}\left[N_{\lambda}^{v}(t)\right]}-1\right|\right] \leq 2
$$

Therefore, we can apply the dominated convergence theorem and calculate the following limit:

$$
\lim _{\lambda \rightarrow+\infty} \mathbb{E}\left[\left|\frac{N_{\lambda}^{v}(t)}{\mathbb{E}\left[N_{\lambda}^{v}(t)\right]}-1\right|\right]=\lim _{\lambda \rightarrow+\infty} \sum_{j=0}^{+\infty}\left|\frac{j}{\frac{\lambda t^{v}}{\Gamma(v+1)}}-1\right|\left(\lambda t^{v}\right)^{j} E_{v, j v+1}^{j+1}\left(-\lambda t^{v}\right) .
$$

Taking account of the behavior of the generalized Mittag-Leffler function for large $z$ (see Saxena et al. (2004) for details), i.e.:

$$
E_{\alpha, \beta}^{\delta}(z) \sim \mathscr{O}\left(|z|^{-\delta}\right), \quad|z|>1,
$$

we can conclude that limit (4.1) equals 0 . This fact proves the result since convergence in mean implies convergence in probability.

The previous result can be extended to a more general setting. Recalling the expression (1.10) for the moments of $N_{\lambda}^{v}(t)$, the proof of the next proposition is similar to that of Proposition 4.1 and thus is omitted.

Proposition 4.2. Let $v \in(0,1]$ and $r \in \mathbb{N}$. Then, for a fixed $t>0$,

$$
\frac{\left[N_{\lambda}^{v}(t)\right]^{r}}{\mathbb{E}\left\{\left[N_{\lambda}^{v}(t)\right]^{r}\right\}} \underset{\lambda \rightarrow+\infty}{\stackrel{\text { Prob }}{\longrightarrow}} 1 \text {. }
$$

In order to prove an analogous result for $M^{v}(t)$, in the following lemma we give a formal expression for the moments of such a process.

Lemma 4.1. The $m^{\text {th }}$ order moment of the process $M^{v}(t), t \geq 0$, reads

$$
\begin{aligned}
\mathbb{E}\left\{\left[M^{v}(t)\right]^{m}\right\}= & \sum_{r=0}^{m} \frac{t^{r v}}{\Gamma(r v+1)} \sum_{i_{1}+\ldots+i_{k}=r}\left(\begin{array}{c}
r \\
i_{1}, \ldots, i_{k}
\end{array}\right) \lambda_{1}^{i_{1}} \ldots \lambda_{k}^{i_{k}} \\
& \times\left.\sum_{n_{1}+\ldots+n_{k}=m}\left(\begin{array}{c}
m \\
n_{1}, \ldots, n_{k}
\end{array}\right)\left[\frac{d^{n_{1}}}{d s^{n_{1}}}\left(e^{s}-1\right)^{i_{1}} \ldots \frac{d^{n_{k}}}{d s^{n_{k}}}\left(e^{k s}-1\right)^{i_{k}}\right]\right|_{s=0} .
\end{aligned}
$$

Proof: By applying Hoppe's formula in order to evaluate the derivatives of the moment generating function of the process $M^{v}(t)$, cf. (2.5), we have

$$
\mathbb{E}\left\{\left[M^{v}(t)\right]^{m}\right\}=\left.\sum_{r=0}^{m} \frac{\left.\left(E_{v, 1}(z)\right)^{(r)}\right|_{z=\sum_{j=1}^{k} \lambda_{j}\left(e^{j s}-1\right) t^{v}}}{r !} A_{m, r}\left(\sum_{j=1}^{k} \lambda_{j}\left(e^{j s}-1\right) t^{v}\right)\right|_{s=0},
$$

where

$$
\begin{aligned}
A_{m, r}\left(\sum_{j=1}^{k} \lambda_{j}\left(e^{j s}-1\right) t^{v}\right)= & \sum_{h=0}^{r}\left(\begin{array}{l}
r \\
h
\end{array}\right)\left(-\sum_{j=1}^{k} \lambda_{j}\left(e^{j s}-1\right) t^{v}\right)^{r-h} \\
& \times \frac{d^{m}}{d s^{m}}\left(\sum_{j=1}^{k} \lambda_{j}\left(e^{j s}-1\right) t^{\nu}\right)^{h}
\end{aligned}
$$

Finally, after using rather cumbersome algebra, we obtain (4.2). 
It is now immediate to verify the following result for $M^{v}(t)$.

Proposition 4.3. Let $v \in(0,1]$ and $m \in \mathbb{N}$. Then, for $i \in\{1,2, \ldots, k\}$ and for a fixed $t>0$, we have

$$
\frac{\left[M^{v}(t)\right]^{m}}{\mathbb{E}\left\{\left[M^{v}(t)\right]^{m}\right\}} \underset{\lambda_{i} \rightarrow+\infty}{\stackrel{\text { Prob }}{\longrightarrow}} 1 .
$$

Proof: By virtue of (4.2), convergence in probability can be obtained by proving convergence in mean, as in Proposition 4.1.

The results presented in this section deserve interest in some physical contexts. We recall that a family of random variables $U^{(\lambda)}$ exhibits cut-off behavior at mean times if (see, for instance, Definition 1 of Barrera et al., 2009)

$$
\frac{U^{(\lambda)}}{\mathbb{E}\left[U^{(\lambda)}\right]} \underset{\lambda \rightarrow+\infty}{\stackrel{\text { Prob }}{\longrightarrow}} 1 .
$$

Hence, Propositions 4.1, 4.2 and 4.3 show that the processes $\left[N_{\lambda}^{v}(t)\right]^{m}$ and $\left[M^{v}(t)\right]^{m}$, $m \in \mathbb{N}$, exhibit cut-off behavior at mean times with respect to the relevant parameters or, roughly speaking, that they somehow converge very abruptly to equilibrium.

We finally remark that in this context the sufficient condition given in Proposition 1 of Barrera et al. (2009) is not useful to prove Proposition 4.1, since such condition holds only when $v=1$.

\section{Acknowledgements}

The authors would like to thank an anonymous referee for some useful comments.

\section{References}

R. M. Adelson. Compound poisson distributions. Oper. Res. Soc. 17 (1), $73-75$ (1966). DOI: $10.2307 / 3007241$.

M. Alipour, L. Beghin and D. Rostamy. Generalized fractional nonlinear birth processes. Methodol. Comput. Appl. Probab. 17 (3), 525-540 (2015). MR3377847.

J. Barrera, O. Bertoncini and R. Fernández. Abrupt convergence and escape behavior for birth and death chains. J. Stat. Phys. 137 (4), 595-623 (2009). MR2565098.

L. Beghin and C. Macci. Alternative forms of compound fractional Poisson processes. Abstr. Appl. Anal. pages 747503, 30 (2012). MR2991021.

L. Beghin and C. Macci. Fractional discrete processes: compound and mixed Poisson representations. J. Appl. Probab. 51 (1), 19-36 (2014). MR3189439.

L. Beghin and C. Macci. Multivariate fractional poisson processes and compound sums (2016+). To appear in Adv. in Appl. Probab.

L. Beghin and E. Orsingher. Fractional Poisson processes and related planar random motions. Electron. J. Probab. 14, no. 61, 1790-1827 (2009). MR2535014.

L. Beghin and E. Orsingher. Poisson-type processes governed by fractional and higherorder recursive differential equations. Electron. J. Probab. 15, no. 22, 684-709 (2010). MR2650778.

V. E. Bening and V. Y. Korolev. Generalized poisson models and their applications in insurance and finance. Modern Probability and Statistics. Utrecht ; Boston : VSP (2002). ISBN 90-6764-366-1. 
R. Biard and B. Saussereau. Fractional Poisson process: long-range dependence and applications in ruin theory. J. Appl. Probab. 51 (3), 727-740 (2014). MR3256223.

A. Di Crescenzo, B. Martinucci and A. Meoli. Fractional growth process with two kinds of jumps. Computer Aided Systems Theory - EUROCAST 2015: 15th International Conference, pages 158-165. Springer International Publishing (2015). ISBN 978-3319-27340-2. DOI: 10.1007/978-3-319-27340-2_21.

R. Gorenflo and F. Mainardi. Laplace-Laplace analysis of the fractional Poisson process. AMADE. Papers and memoirs to the memory of Prof. Anatoly Kilbas, pages 43-58. Publishing House of BSU, Minsk (2012).

R. Gorenflo and F. Mainardi. On the fractional poisson process and the discretized stable subordinator. ArXiv Mathematics e-prints (2013). arXiv: 1305.3074v1.

A. Kumar, E. Nane and P. Vellaisamy. Time-changed Poisson processes. Statist. Probab. Lett. 81 (12), 1899-1910 (2011). MR2845907.

N. Laskin. Fractional Poisson process. Commun. Nonlinear Sci. Numer. Simul. 8 (3-4), 201-213 (2003). Chaotic transport and complexity in classical and quantum dynamics. MR2007003.

N. Laskin. Some applications of the fractional Poisson probability distribution. J. Math. Phys. 50 (11), 113513, 12 (2009). MR2567219.

A. Maheshwari and P. Vellaisamy. On the long-range dependence of fractional poisson and negative binomial processes (2016+). To appear in J. Appl. Probab.

F. Mainardi. Fractional relaxation-oscillation and fractional diffusion-wave phenomena. Chaos Solitons Fractals 7 (9), 1461-1477 (1996a). MR1409912.

F. Mainardi. The fundamental solutions for the fractional diffusion-wave equation. Appl. Math. Lett. 9 (6), 23-28 (1996b). MR1419811.

F. Mainardi, R. Gorenflo and E. Scalas. A fractional generalization of the Poisson processes. Vietnam J. Math. 32 (Special Issue), 53-64 (2004). MR2120631.

A. M. Mathai and H. J. Haubold. Special Functions for Applied Scientists. Springer-Verlag New York (2008). ISBN 978-0-387-75893-0. DOI: 10.1007/978-0-387-75894-7.

M. M. Meerschaert, E. Nane and P. Vellaisamy. The fractional Poisson process and the inverse stable subordinator. Electron. J. Probab. 16, no. 59, 1600-1620 (2011). MR2835248.

E. Orsingher. Fractional Poisson processes. Sci. Math. Jpn. 76 (1), 139-145 (2013). MR3099209.

E. Orsingher and L. Beghin. Time-fractional telegraph equations and telegraph processes with Brownian time. Probab. Theory Related Fields 128 (1), 141-160 (2004). MR2027298.

E. Orsingher and F. Polito. The space-fractional Poisson process. Statist. Probab. Lett. 82 (4), 852-858 (2012). MR2899530.

E. Orsingher and F. Polito. On the integral of fractional Poisson processes. Statist. Probab. Lett. 83 (4), 1006-1017 (2013). MR3041370.

E. Orsingher and B. Toaldo. Counting processes with Bernštein intertimes and random jumps. J. Appl. Probab. 52 (4), 1028-1044 (2015). MR3439170.

M. Politi, T. Kaizoji and E. Scalas. Full characterization of the fractional poisson process. Europhysics Letters 96 (2), 20004 (2011). DOI: 10.1209/0295-5075/96/20004.

F. Polito and E. Scalas. A generalization of the space-fractional poisson process and its connection to some Lévy processes. ArXiv Mathematics e-prints (2016). arXiv: $1502.03115 \mathrm{v} 3$. 
T. R. Prabhakar. A singular integral equation with a generalized Mittag Leffler function in the kernel. Yokohama Math. J. 19, 7-15 (1971). MR0293349.

R. K. Saxena, A. M. Mathai and H. J. Haubold. Unified fractional kinetic equation and a fractional diffusion equation. Astrophysics and Space Science 290 (3), 299-310 (2004). DOI: 10.1023/B:ASTR.0000032531.46639.a7.

E. Scalas. A class of CTRWs: compound fractional Poisson processes. In Fractional dynamics, pages 353-374. World Sci. Publ., Hackensack, NJ (2012). MR2932614. 